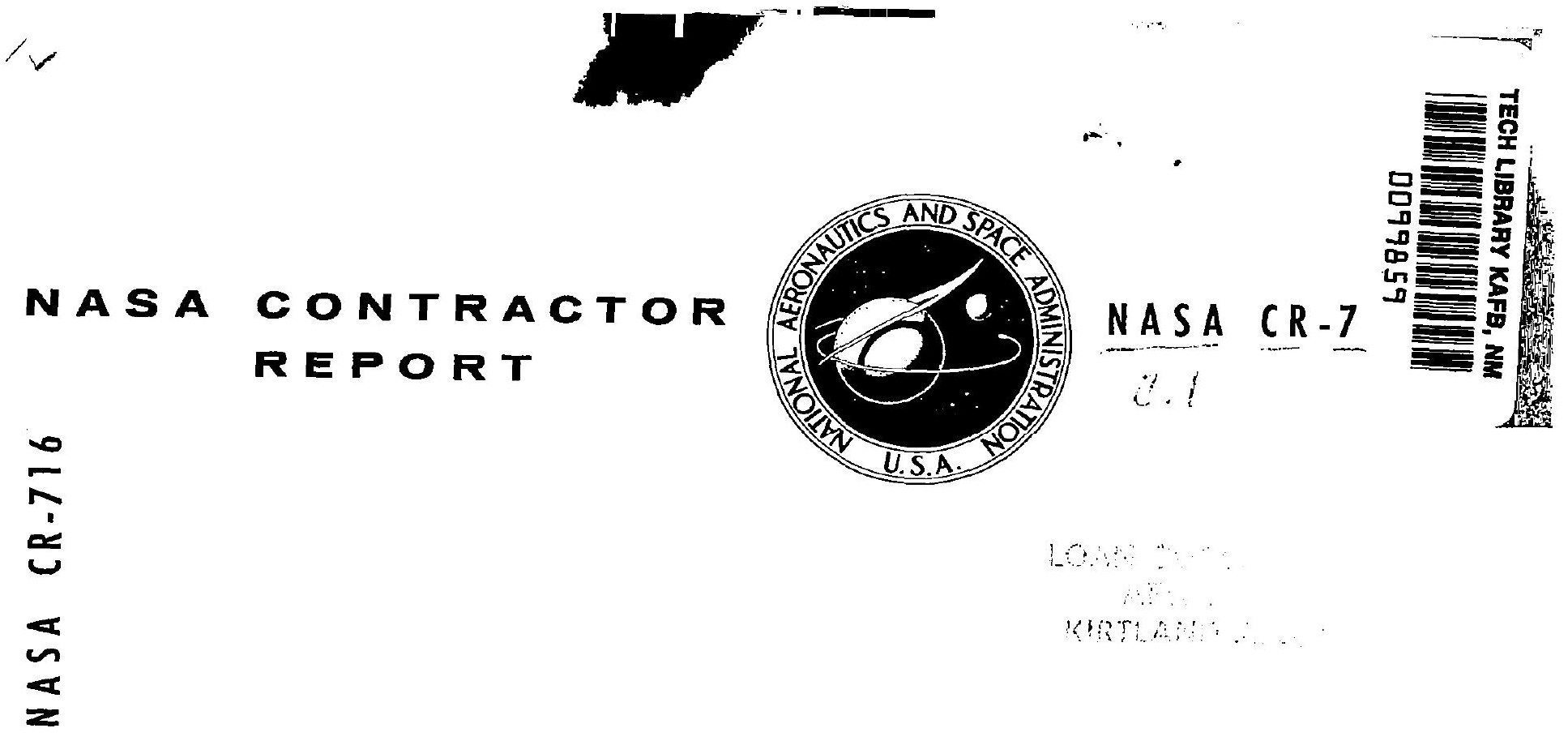

\title{
CONTROL OF NONLINEAR MULTIVARIABLE SYSTEMS
}

\section{by D. P. Lindorff}

Prepared by

UNIVERSITY OF CONNECTICUT

Storrs, Conn.

for

NATIONAL AERONAUTICS AND SPACE ADMINISTRATION - WASHINGTON, D. C. - FEBRUARY 1967 
NASA CR-716

CONTROL OF NONLINEAR MULTIVARIABLE SYSTEMS

By D. P. Lindorff

Distribution of this report is provided in the interest of information exchange. Responsibility for the contents resides in the author or organization that prepared it.

Prepared under Grant No. NsG-309 by UNIVERSITY OF CONNECTICUT

Storrs, Conn.

for

NATIONAL AERONAUTICS AND SPACE ADMINISTRATION

For sale by the Clearinghouse for Federal Scientific and Technical Information Springfield, Virginia 22151 - Price $\$ 1.00$ 
CONTROL OF NONLINEAR MULTIVARIABLE SYSTEMS

\author{
D. P. Lindorff \\ Department of Electrical Engineering \\ University of Connecticut \\ Storrs, Connecticut 06268
}

\title{
I. INTRODUCTION
}

A synthesis technique is developed which has application to nonlinear, nonautonomous multivariable systems. The control concept, which is rooted in Liapunov's Direct Method, has been described in a number of recent papers $1,2,3$ In this paper the method is extended so as to be applicable to nonlinear plants with inequality constraints on the controlled forces. Referring to Fig. 1, the basic control configuration is shown in which a multivariable plant is caused to track a model through the action of a fixed-law controller. The design of the controller is to be such as to cause the error (e) to be bounded. A design example concerned with the control of an exothermic chemical reactor is used to illustrate an application of the technique.

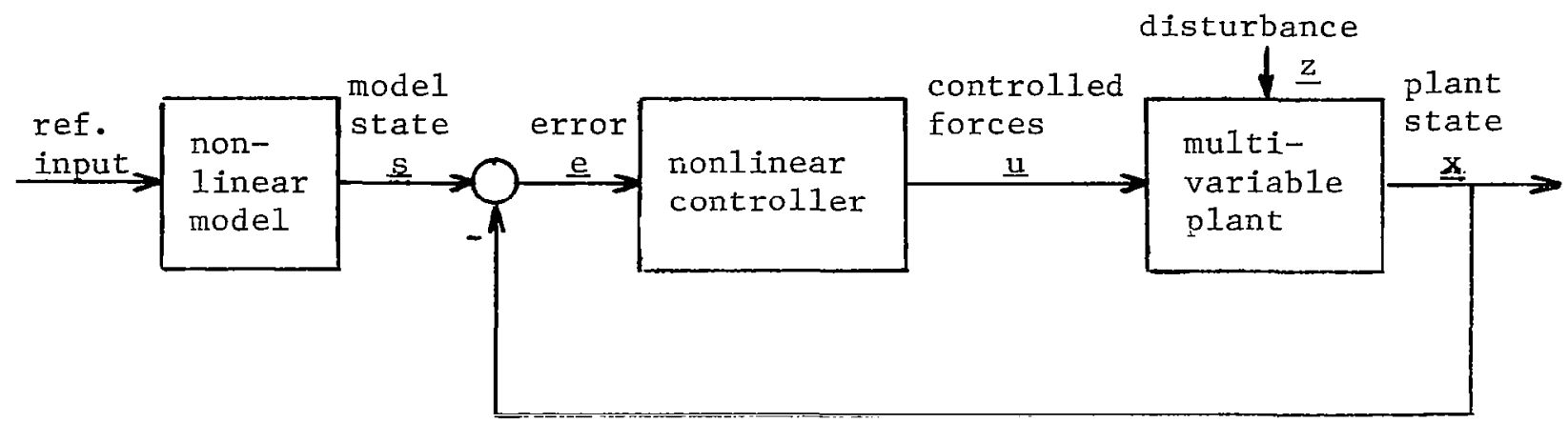

Fig. 1. Basic Control Configuration

Grayson ${ }^{1}$ and Monopoli ${ }^{2}$ have developed a single-input single-output modeltracking system in which a time-variable plant is caused to track a linear, stationary model. Lindorff and Monopoli ${ }^{3}$ have applied the model-tracking scheme to a limited class of multivariable plants. In the present paper, the multivariable problem is developed further, so as to be applicable to a more general class of nonlinear systems with inequality constraints on the control forces. With given limits on the amplitudes of the controlled forces, a region of the plant state space can be found in which the control law is valid, the size of this region being a function of ccrtain of the model states. A novel feature of the method relates to nonlinear constraints which may be placed on the model to guarantee validity of the control law within a specified region of the state space. 


\section{DESCRIPTION OF THE MULTIVARIABLE PLANT}

A multivariable process contains a multiplicity of inputs and outputs. If the outputs are to be usefully controlled, it is necessary that each output can be influenced by the controlled inputs. If the control forces are limited in amplitude, the region of the state space in which this control can be accomplished will also be limited. It will be assumed throughout that each output can be identified with a forcing function so that within some region of the state space each output can be independently controlled. It is appropriate therefore to require that the number of controlled inputs be equal to the number of outputs.

The plant is assumed to be described by $\underset{*}{z}$ set of $\mathbf{n}$ first-order ordinary differential equations having the vector form

$$
\underline{\dot{x}}=g(\underline{x}, \underline{z}, t)+\Phi(\underline{x}, t) \underline{u}(t),
$$

and a set of $q$ equations defining the plant outputs, these having the vector form

$$
\underline{\mathrm{v}}=\mathrm{C} \underline{\mathrm{x}} .
$$

In the above equations, it is assumed that

$\underline{x}$ is an $n$ dimensional state vector,

$\underline{v}$ is a $q$ dimensional output vector $(q \leq n)$,

$\underline{z}$ is a q dimensional disturbance vector,

$\mathrm{g}$ is an $\mathrm{n}$ dimensional vector function,

$\underline{u}$ is a q dimensional controlled-force vector,

$\Phi$ is an $\mathrm{n} x \mathrm{q}$ matrix containing no time-derivative operators,

$\mathrm{C}$ is a constant $\mathrm{q} \times \mathrm{n}$ matrix.

(2.1) is assumed to satisfy conditions for existence and uniqueness of solution in some region $R_{0}$ in $(\underline{x}, t)$ space.

It will be assumed that (2.1) is written in partitioned normal form. This means that if $x$ is partitioned into $q$ subvectors $\underline{x}_{I}, \ldots, \underline{x}_{q}$, so that (2.1) can be written as

$$
\left.\begin{array}{c}
\dot{\underline{x}}_{1}=g_{1}+\underline{\Phi}_{1} \underline{u}_{1} \\
\vdots \\
\dot{x}_{q}=g_{q}+\Phi_{q} \underline{u}_{q}
\end{array}\right\}
$$

then each of these equations is to be written in normal form. Explicitly, if $\underline{x}_{i}$ has normal components $\left(x_{1}^{1}, \ldots, x_{m}^{1}\right)$, then $x_{1}^{i}=v_{i}$, and

* The notation $\dot{x}$ denotes the time derivative, $\mathrm{dx} / \mathrm{dt}$. The functional dependence on $\underline{z}$ will sometimes be suppressed for simplicity of notation. 


$$
\left[\begin{array}{c}
\dot{x}_{1}^{i} \\
\dot{x}_{2}^{i} \\
\vdots \\
\dot{x}_{m-1}^{i} \\
\dot{x}_{m}^{i}
\end{array}\right]=\left[\begin{array}{c}
x_{2}^{i} \\
x_{3}^{i} \\
\vdots \\
x_{m}^{i} \\
g_{m}^{i}
\end{array}\right]+\left[\begin{array}{ccc}
0 & \ldots & 0 \\
0 & \ldots & 0 \\
\vdots & & \vdots \\
0 & & 0 \\
\phi_{m 1}^{i} & \cdots & \phi_{m q}^{i}
\end{array}\right]\left[\begin{array}{c}
u_{1} \\
u_{2} \\
\vdots \\
u_{q-1} \\
u_{q}
\end{array}\right] .
$$

It is permitted that $g_{m}^{i}=g_{m}^{i}(\underline{x}, \underline{z}, t)$, and $\phi_{m j}^{i}=\phi_{m j}^{i}(\underline{x}, t), i=1, \ldots, q$.

The normal form is important to future developments for a number of reasons to be explained subsequently. A direct consequence of the normal form is that zeros are forced to appear in all but the last row of the $\Phi$ matrix of (2.4). This fact is used advantageously in deriving a workable control law.

\section{DEVELOPMENT OF SYNTHESIS TECHNIQUE}

With reference to Fig. 1, a synthesis technique is developed which

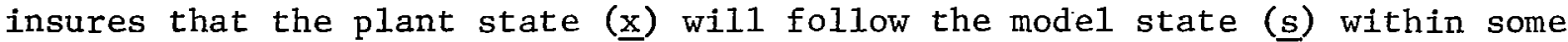
bound of error. The problem may be formally stated as follows:

Given a set of inequality constraints on the elements of the control vector ( $\underline{u})$ and certain of the elements of the model state vector ( $\underline{\text { s }}$ ), find a region $R$ in $x$ space inside which a control law can be established so that the error (e ) will be held within some bound.

To solve this problem, the coordinates of the plant state vector ( $\underline{x}$ ) are first transformed to the error coordinates (e) by the equation

$$
\underline{\mathrm{e}}=\underline{\mathrm{s}}-\underline{\mathrm{x}},
$$

where it is understood that the partitioned forms of $x$ and $\underline{s}$ are similar. Thus, for each subvector $\underline{s}_{i}$ with elements $\left(x \dot{1}, \ldots, x_{m}^{\frac{1}{1}}\right)$, there is a subvector $\underline{s}_{i}$ with elements $\left(s \frac{i}{1}, \ldots, s_{m}^{i}\right)$ defined according to $\dot{s}_{1}^{i}=s_{2}^{i}, \ldots, \dot{s}_{m-1}^{i}=s_{m}^{i}$, the element $s_{1}^{i}$ being defined as the desired value of the plant output $v_{i}$.

Using (2.1) and (3.1), it follows that the differential equation of the error is given by

$$
\underline{\dot{e}}=-\underline{h}(\underline{x}, t)
$$

where

$$
\underline{h}=-\underline{\dot{s}}(t)+g(\underline{x}, t)+\Phi(\underline{x}, t) \underline{u}(t) \text {. }
$$

In partitioned form (3.3) can be expressed as 


$$
\underline{\dot{e}}_{i}=-\underline{h}_{i}(\underline{x}, t), i=1, \ldots, q \text {. }
$$

The control objective is to manipulate $\underline{u}$ within its constraints so as to keep e in the proximity of the point $\underline{e}=\underline{0}$. This can be accomplished if, for some positive-definite function $V(\underline{e})$, and with respect to (3.2), the algebraic sign of $\mathrm{dV} / \mathrm{dt}$ is caused to be negative outside of some suitably small region about the origin. A difficulty in attempting to shrink this region to zero has been pointed out, notably by Monopoli ${ }^{4}$. This difficulty stems from the fact that $\underline{u}(t)$ may be implemented in such a way that an equilibrium solution $\underline{e}=\underline{0}$ does not exist as the size of this region shrinks to zero. It will be seen that this is the case in the present paper. Hence, although the synthesis procedure is motivated by Liapunov's Direct Method, the solution does not fall within the realm of Liapunov theory, since boundedness rather than stability must be used as a design criterion.

In order to find a suitable function $\mathrm{V}(\mathrm{e})$, use will be made of the theorem attributed to Liapunov which states ${ }^{5}$ :

If and only if $A$ is a stability matrix, i.e., such that al1 $\lambda$ roots of the determintal equation $|A-\lambda I|=0$ have negative real parts, then for any positive-definite symmetric matrix $Q$, there is a positive-definite symmetric matrix $P$ which is a unique solution to the equation*

$$
-Q=A^{t} P+P A \text {. }
$$

In the synthesis procedure to be developed, the A matrix need bear no relationship to the plant or the model. Hence, we are free to stipulate that A be partitioned in the diagonal form,

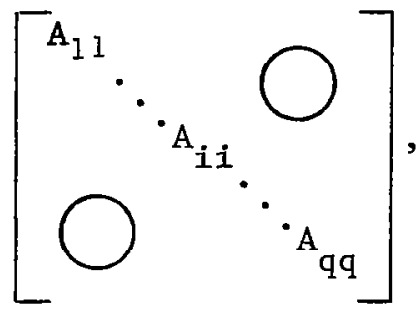

so that $A$ and $\underline{e}$ are conformably related. Thus, if a subvector $\underline{e}_{i}$ is of $\mathrm{m}$ dimensions, then $A_{i i}$ is an $m \times m$ submatrix, A being an $n \times n$ matrix.

Given any symmetric positive-definite $Q$ matrix which is partitioned in a diagonal form similar to (3.6) so that $A$ and $Q$ are conformably partitioned, it has been shown ${ }^{3}$ that $P$, as a solution to (3.5), will be similarly partitioned in diagonal form. We have, therefore, established that $Q$ and $P$ can be found such that both

${ }^{*}{ }^{t}$ denotes the transpose of $A$. 


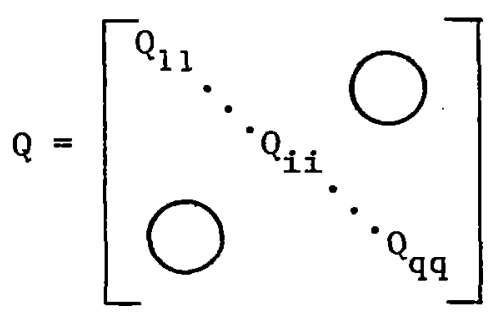

and

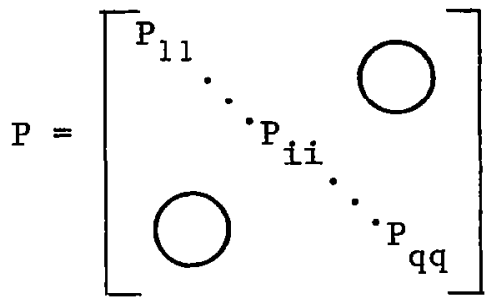

are positive definite.

In order to make use of this result, (3.2) will be written in the form

$$
\underline{\dot{e}}=-\underline{h}(\underline{x}, t)-A \underline{e}+A \underline{e} \text {. }
$$

In compact notation let (3.9) be written as

$$
\underline{\dot{e}}=-\underline{f}(\underline{x}, t)+A \underline{e} .
$$

Writing $\underline{f}$ as an explicit function of $x, t$, accounts for its dependence on $\underline{e}$ because $\underline{\underline{e}}=\underline{e}(\underline{x}, t)$.

We turn now to the positive-definite quadratic form

$$
V=\underline{e}^{t} \underline{P},
$$

and its time derivative

$$
\dot{\mathrm{V}}=\underline{\dot{e}}^{t} \underline{\mathrm{e}}+\underline{e}^{\mathrm{t}} \underline{\dot{e}} \dot{\mathrm{e}} .
$$

Substituting (3.10) into (3.12), it follows by using the symmetry of $P$ that

$$
\dot{V}=\underline{e}^{t}\left(A^{t} P+P A\right) \underline{e}-2 \underline{e}^{t} \underline{\underline{f}}(\underline{x}, t) \text {. }
$$

Substituting (3.5) into (3.13),

$$
\dot{\mathrm{V}}=-\underline{e}^{t} \underline{\underline{e}}-2 \underline{e}^{t} \operatorname{Pf}(\underline{x}, t) \text {. }
$$

Since $Q$ is positive definite, it follows for $\underline{e} \neq \underline{0}$ that $\dot{V}$ will be negative if

$$
\underline{e}^{t} \underline{P} \underline{f}(\underline{x}, t) \geq 0
$$


Since $f$ is a function of the controlled forces $\underline{u}$, it is reasonable to expect that the inequality in (3.15) can, at least to some extent, be controlled by $\underline{u}(t)$.

Turning now to (3.8) and (3.11), it is observed that $V$ can be expressed in the form

$$
v=\sum_{i=1}^{q} v_{i}\left(\underline{e}_{i}\right)
$$

wherein

$$
v_{i}=e_{i}^{t} p_{i i} \underline{e}_{i}, i=1, \ldots, q
$$

Since $V$ is positive definite, then each $V_{i}$ is also positive definite as a consequence of the diagonal partitioned form of $P$.

Simarly (3.14) can be written in the form

$$
\dot{V}=\sum_{i=1}^{q} \dot{V}_{i}
$$

wherein

$$
\dot{\mathrm{V}}_{i}=-\underline{e}_{i}^{t} \mathrm{Q}_{i} \underline{e}_{i}-2 \underline{e}_{i}^{t} \mathrm{P}_{i i} \underline{f}_{i}(\underline{x}, t), i=1, \ldots, q .
$$

A sufficient condition for boundedness of the solution to (3.2) can now be obtained by controlling the sign of each $V_{i}$ and thereby establishing the boundedness of each subvector $\underline{e}_{i}$, for $i=1, \ldots, q$.

To perceive the way in which $u_{i}$ can be used to control the sign of $\dot{V}_{i}$, an explicit expression for the term $e_{j}^{t^{i}} \mathrm{P}_{i i} \underline{f}_{i}$ in (3.19) is required. It will be helpful first to consider the following example:

Example 3.1:

Let (2.1) take on the explicit form

$$
\begin{aligned}
& \dot{x}_{1}^{1}=x_{2}^{1} \\
& \dot{x}_{2}^{1}=g_{2}^{1}(\underline{x})+u_{1}(t) \\
& \dot{x}_{1}^{2}=x_{2}^{2} \\
& \dot{x}_{2}^{2}=g_{2}^{2}(\underline{x})+u_{1}(t)+u_{2}(t)
\end{aligned}
$$

where it is understood that the plant outputs are defined by $v_{1}=x_{1}^{1}, v_{2}=x_{1}^{2}$, and 


$$
\Phi=\left|\begin{array}{cc}
0 & 0 \\
1 & 0 \\
-0 & 0 \\
1 & 1
\end{array}\right|
$$

Equation (3.2) now has the explicit form

$$
\begin{aligned}
& \dot{\mathrm{e}}_{1}^{1}=\mathrm{e}_{2}^{1} \\
& \dot{\mathrm{e}}_{2}^{1}=\dot{\mathrm{s}}_{2}^{1}-\mathrm{g}_{2}^{1} \underline{(\underline{x})}-\mathrm{u}_{1} \\
& \dot{\mathrm{e}}_{1}^{2}=\mathrm{e}_{2}^{2} \\
& \dot{\mathrm{e}}_{2}^{2}=\dot{\mathrm{s}}_{2}^{2}-\mathrm{g}_{2}^{2}(\underline{x})-\mathrm{u}_{1}-\mathrm{u}_{2}
\end{aligned}
$$

If the A matrix is partitioned according to (3.6), then

$$
A=\left[\begin{array}{c:c}
A_{11} & 0 \\
\hdashline 0 & A_{22}
\end{array}\right] \text {. }
$$

We are free to impose the requirement that each $A_{i i}$ be expressed in the normal form, so that

$$
A_{i i}=\left[\begin{array}{cc}
0 & 1 \\
a_{21}^{i} & a_{22}^{i}
\end{array}\right], i=1,2 .
$$

Then according to (3.9), (3.24), it is seen that (3.22) can be written as

$$
\begin{aligned}
\dot{\mathrm{e}}_{1}^{1}= & \mathrm{e}_{2}^{1} \\
\dot{\mathrm{e}}_{2}^{1}= & \dot{s}_{2}^{1}-\mathrm{g}_{2}^{1}(\underline{\mathrm{x}})-\mathrm{u}_{1}-\left(\mathrm{a}_{21}^{1} \mathrm{e}_{1}^{1}+a_{22}^{1} \mathrm{e}_{2}^{1}\right)+\left(a_{21}^{1} e_{1}^{1}+a_{22}^{1} \mathrm{e}_{2}^{1}\right) \\
\dot{\mathrm{e}}_{1}^{2}= & \mathrm{e}_{2}^{2} \\
\dot{\mathrm{e}}_{2}^{2}= & \dot{\mathrm{s}}_{2}^{2}-\mathrm{g}_{2}^{2}(\underline{\mathrm{x}})-\mathrm{u}_{1}-\mathrm{u}_{2}-\left(\mathrm{a}_{21}^{2} \mathrm{e}_{1}^{2}+\mathrm{a}_{22}^{2} \mathrm{e}_{2}^{2}\right) \\
& +\left(\mathrm{a}_{21}^{2} \mathrm{e}_{1}^{2}+\mathrm{a}_{22}^{2} \mathrm{e}_{2}^{2}\right)
\end{aligned}
$$

We can express (3.25) conveniently in terms of the compact form of (3.10). Thus, using subvector notation,

$$
\left[\begin{array}{l}
\dot{e}_{1} \\
\dot{e}_{2}
\end{array}\right]=-\left[\begin{array}{l}
\underline{f}_{1} \\
\underline{f}_{2}
\end{array}\right]+\left[\begin{array}{c:c}
A_{11} & 0 \\
\hdashline 0 & - \\
0 & A_{22}
\end{array}\right]\left[\begin{array}{l}
\underline{e}_{1} \\
\underline{e}_{2}
\end{array}\right] \text {, }
$$


it follows that

$$
\begin{aligned}
& \underline{f}_{1}=\left[\begin{array}{c}
0 \\
-\dot{s}_{2}^{1}+g_{2}^{1}(\underline{x})+a_{21}^{1} e_{1}^{1}+a_{22}^{1} e_{2}^{1}+u_{1}
\end{array}\right] \\
& \underline{f}_{2}=\left[\begin{array}{c}
0 \\
-\dot{s}_{2}^{2}+g_{2}^{2}(\underline{x})+a_{21}^{2} e_{1}^{2}+a_{22}^{2} e_{2}^{2}+u_{1}+u_{2}
\end{array}\right] .
\end{aligned}
$$

Assuming that the matrix $P$ is in the form

$$
P=\left[\begin{array}{c:c}
\mathrm{P}_{11} & 0 \\
\hdashline 0 & \mathrm{P}_{22}
\end{array}\right],
$$

with

$$
P_{i i}=\left[\begin{array}{cc}
p_{11}^{i} & p_{12}^{i} \\
p_{21}^{i} & p_{22}^{i}
\end{array}\right], i=1,2
$$

then with reference to (3.19), we can state for this example that

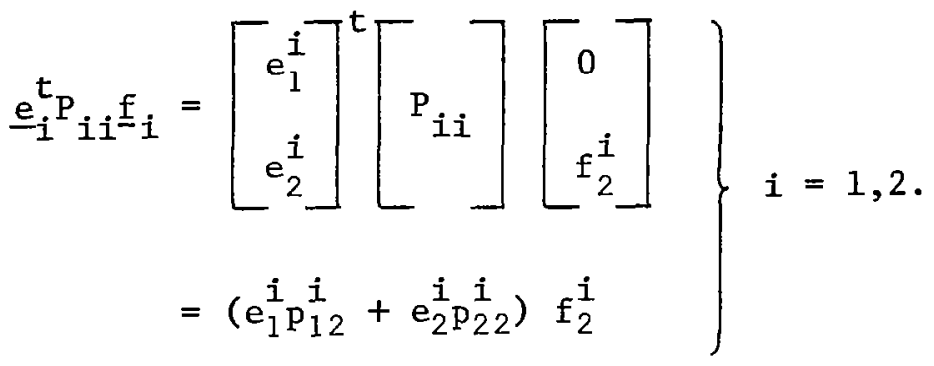

The fact that $f \frac{i}{2}$ is a factorable term in this equation is a direct consequence of having (3.25) in partitioned normal form.

The results of this example are readily extended to the general case. Thus, as a generalization of (3.29), we have

$$
e_{i}^{t} p_{i i} \underline{f}_{i}=\gamma_{i} f_{m}^{i}, \quad i=1, \ldots, q,
$$

where

$$
\gamma_{i}=\sum_{j=1}^{m} e_{j}^{i} p_{j m}^{i} .
$$




\section{Furthermore}

$$
\underline{\mathrm{f}}_{\mathrm{i}}=\left[\begin{array}{c}
0 \\
\vdots \\
0 \\
\mathrm{f} \\
\mathrm{m}
\end{array}\right]
$$

wherein

$$
f_{m}^{i}=\xi_{1}(\underline{x}, t)+\phi_{m i}(\underline{x}, t) u_{i},
$$

and

$$
\xi_{i}=-\dot{s}_{m}^{i}+g_{m}^{i}(\underline{x}, t)+\sum_{j=1}^{m} a_{m j}^{i} e_{j}^{i}+\underset{\substack{j=1 \\ j \neq i}}{q} \phi_{m j}(x, t) u_{j} .
$$

To require in (3.30) that

$$
\underline{e}_{i}^{t} P_{i i} \underline{f}_{i} \geq 0
$$

is to require that

$$
\operatorname{sgn}\left(f_{m}^{i}\right)=\operatorname{sgn}\left(\gamma_{i}\right)
$$

where $\operatorname{sgn}($.$) is the sign function portrayed in Fig. 2$.

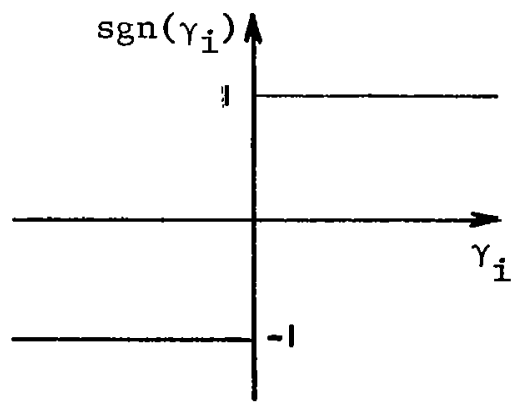

Fig. 2. Plot of $\operatorname{sgn}\left(\gamma_{i}\right)$ vs. $\gamma_{i}$

It can be seen from (3.30) and (3.32) that (3.34) will be valid if two conditions are satisfied, namely:

$$
\left|u_{1}\right| \geq\left|\frac{\xi_{i}(x, t)}{\phi_{i i}(\underline{x}, t)}\right|, \phi_{i i}>0,
$$


and

$$
\frac{u_{i}}{\left|u_{i}\right|}=\operatorname{sgn}\left(\gamma_{i}(t)\right) \text {. }
$$

(3.35) and (3.36) form the basis for a design approach.

In this paper the function $u_{i}$ will be represented as the output of a relay. It will be assumed for each $u_{i}$, that

$$
\left.\begin{array}{rl}
u_{i}=L_{i}, & \gamma_{i} \geq \delta_{i} \\
0 \leq\left|u_{i}\right| \leq L_{i}, & \left|\gamma_{i}\right|<\delta_{i} \\
u_{i}=-L_{i}, & \gamma_{i} \leq \delta_{i}
\end{array}\right\} \quad i=1, \ldots, q,
$$

where $\mathrm{L}_{i}$ and $\delta_{i}$ are positive numbers. Two of the many possible graphical representations of $u_{i}$ versus $\gamma_{i}$ are shown in Fig. 3.
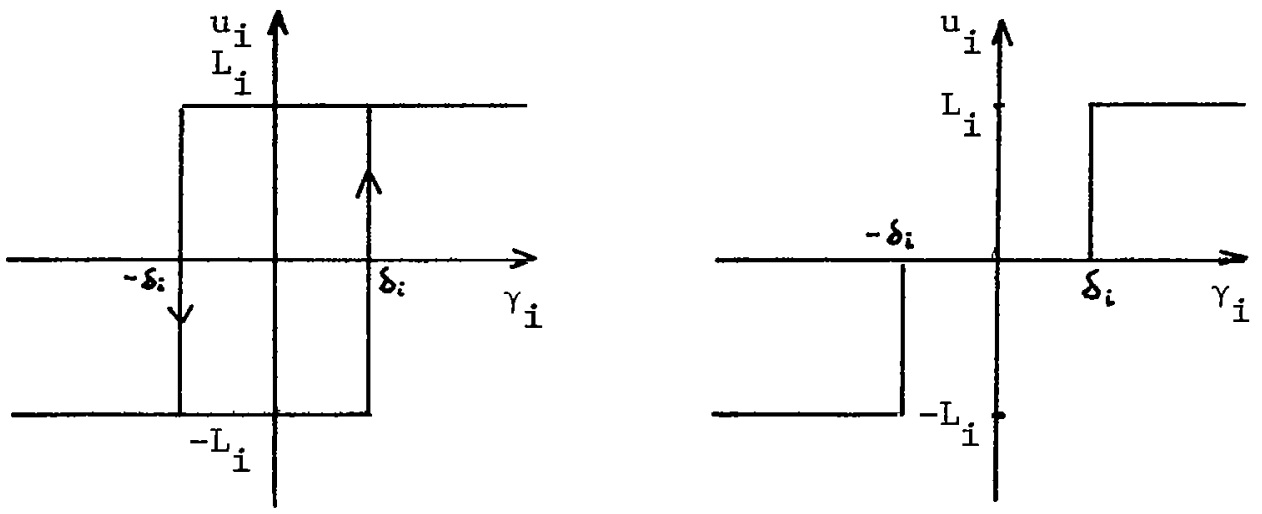

Fig. 3. Two Possible Representations of $u_{i}$ vs. $\gamma_{i}$

For the practical case in which $\delta_{i}>0$, the description of $u_{i}$ in (3.37) fails to guarantee that (3.36) will be satisfied when $\left|\gamma_{i}\right|<\delta_{i}$. In Section IV it will be shown, as a consequence of this fact, that $V_{i}<0$ can be assured only outside a finite region about $e_{j}=\underline{0}$, and that $e_{i}$ has an ultimate bound which becomes arbitrarily small as $\overline{\delta_{i}} \rightarrow \overrightarrow{0}$. From (3.37) it also follows that $\dot{V}_{i}<0$ can be assured only within some restricted region $R_{i}(t)$ of the $\underline{x}$ space. This follows from the inability of $\left|u_{i}\right| \leq L_{i}$ to satisfy (3.35) for all $\underline{x}$.

A design procedure will now be developed by examining the functional dependencies of $R_{i}$. From (3.37) it follows that the region $R_{i}$ in $x$ for which 
(3.35) can be satisfied, consists of the values of $x$ for which

$$
L_{i} \geq\left|\frac{\left.\xi_{(x,}, t\right)}{\phi_{\mathrm{mi}}(\underline{x}, t)}\right|
$$

From (3.32) it is observed that $\xi$, and therefore $R_{i}$, are dependent upon a number of variables, some of which are within the designers control. As stated above, the components of $e_{i}$ will be shown to have a controllable bound. From (3.37) the components of $\underline{u}$ are bounded. Within some region $R_{0}$ it is understood that $\mathrm{g}_{\mathrm{m}}$ and $\phi_{\mathrm{m} j}, i=1, \ldots, \mathrm{q}$, are bounded*. Finally, $\dot{s}_{\mathrm{m}}{ }^{*}$, the time derivative of the highest-order component of the subvector $\underline{s}_{i}$, will be seen to be under the designers control.

The functional dependence of $R_{i}$ on $\dot{s}_{m}^{i}$ points out an important design consideration, namely: For a given level $L_{i}$, the region $R_{i}$ in which the plant is guaranteed of tracking the modci within some bounded error is inversely proportional to bounds on $\dot{s}_{\mathrm{m}}^{i}$. conversely, a model can be designed with constraints on $\dot{s}_{\mathrm{m}}^{i}$ so as to insure that (3.38) will be satisfied within some region $R_{i}$. For multivariable control, if the solution $x(t)$ is restricted to a region $R$, defined as the intersection of the $q$ subregions,

$$
R=\bigcap_{i} R_{i}, \quad i=1, \ldots, q,
$$

then the total error vector $e$ will be within some bound. Clearly, $R_{0}$ includes R.

Finally, it is necessary to define the controlled forces so that (3.5) can be satisfied in some $R_{i}$, for each $i$. This is always possible if a unique set of controlled forces is associated with (2.3) for each value of $i=1$, $\cdots, \mathrm{q}$.

From the preceding discussion, it can be seen that the primary function of the model is to limit the values of $\dot{s}_{m}^{i}$ so that within some region $R$ the tracking error (e-) will be bounded. A constraint on $\dot{s}_{\mathrm{m}}^{\mathbf{i}}$ can be imposed either by means of an on-line computational procedure, to be illustrated in a design example (Section V), or by the use of a limiter, as shown in Fig. 4. In this case, the choice of gains $k_{o}, \ldots, k_{m-1}$, are at the disposal of the designer. The limiting level is the only factor which is related to the synthesis procedure. Furthermore, even though the plant may be controlled so that the components of $\underline{v}$ are essentially noninteracting in response to the model outputs, it does not follow that the model itself need be noninteracting. Therefore, the synthesis procedure allows for an interacting design, should this be desired. A design example illustrates this point. *From $(2.4)$ it is seen that $g_{m}^{i}=g_{m}^{i}(\underline{x}, \underline{z}, t)$. It is assumed that the disturbance
$\underline{z}$ is within known bounds. 


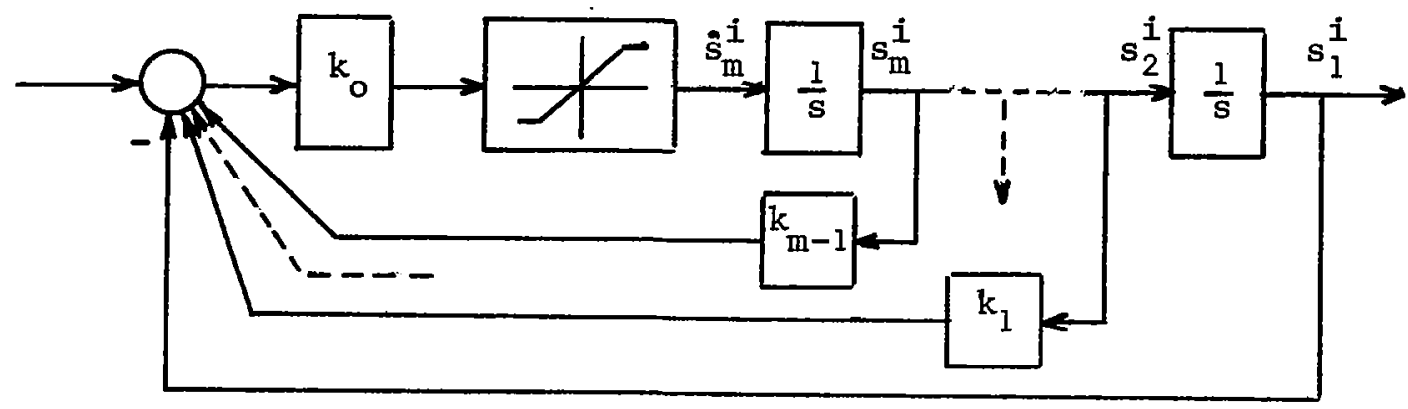

Fig. 4. Nonlinear Model Showing Constraint on $\left|\dot{s}_{\mathrm{m}}^{i}\right|$

IV. BOUNDS ON THE ERROR

For the single-variable system of less than third order, Taylor 6 has found a realistic bound on the error (e). This result can be applied to the multivariable system if each subvector $\left(\underline{e}_{i}\right)$ is less than third order, and if the $\mathrm{P}$ matrix is derived in the diagonal partitioned form of (3.8). For the case in which any $\underline{e}_{i}$ is of higher than second order, the existence of a more conservative bound can be found for which the $P$ matrix need not have the diagonal partitioned form. This result is useful in showing that in the most general case, the bound on the error can be made arbitrarily small as each $\delta_{i}$ in Fig. 3 approaches zero.

The bound found by Taylor will be discussed first, with reference to the multivariable problem. The assumption is made throughout that $x(t)$ lies within the region $R$. For the case in which the $P$ matrix is in the form of (3.8), there is, according to (3.17) for any given $i$, a positive-definite function, $v_{i}$, which can be used to characterize the motion of $e_{i}$. Furthermore, as shown in Fig. 3, if $u_{i}$ is governed by (3.37), then there is a range of $\gamma_{i}$ defined by $\left|\gamma_{i}\right|<\delta_{i}$ for which (3.35), (3.36) are not necessarily satisfied. If $\underline{e}_{i}$ is of second order, the region defined by $\left|\gamma_{i}\right|<\delta_{i}$ lies within a strip in the $\underline{e}_{i}$ plane as shown in Fig. 5 .

Since the components of $e_{i}$ have been defined in normal form, i.e., $\dot{e}_{1}^{i}=e_{2}^{i}$, it follows that motion in the lower half plane must be to the left, whereas motion in the upper half plane must be to the right. For the particular contour $V_{i}=c_{i}$, it follows from a geometrical argument that a trajectory which enters the ellipse $V_{i}=c_{i}$ will remain inside that ellipse. This argument depends upon the interesting observation that points (1), (2) in Fig. 5, representing the intersection of the ellipse with the boundary of the strip, lie on a vertical line. On the other hand, since the control law guarantees $V_{i}<0$ for $\left|\gamma_{i}\right|>\delta_{i}$, it can be said that all trajectories of $e_{i}(t)$ originating outside of $V_{i}=c_{i}$ will ultimately converge on $v_{i}=c_{i}$. Clearly, this ultimate bound on $\underline{e}_{i}$ exists for each $i=1, \ldots, q$. Therefore, $\underline{e}$ is bounded. 


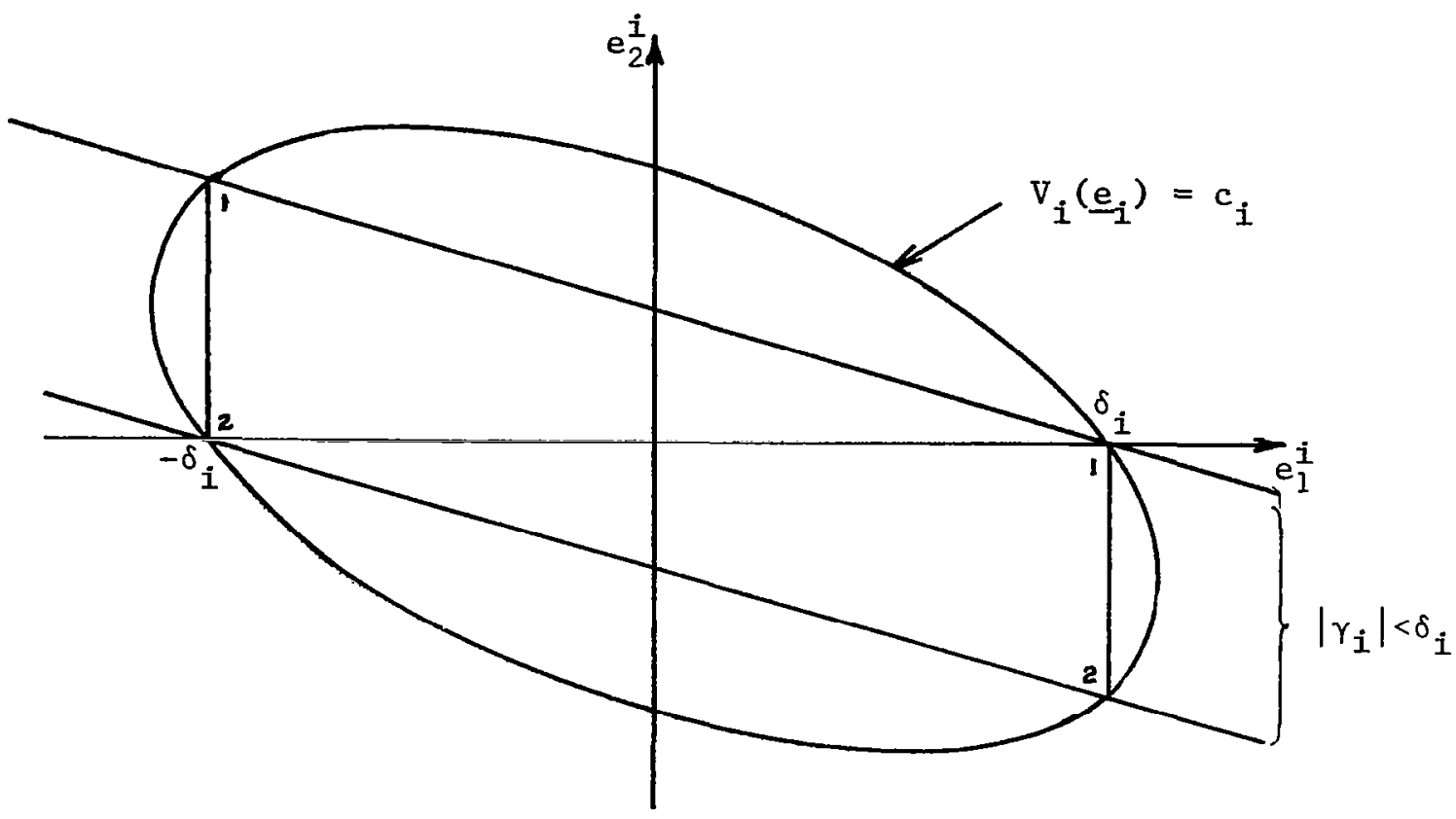

Fig. 5. U1timate Bound for $\underline{e}_{i}$ of Two Dimensions

Turning now to the more general case for which $\underline{e}_{i}$ may be of any order, it is shown in Appendix I that, if each $\delta_{i}$ is small enough and the solution $\underline{x}(t)$ is within $R$, then $\underline{e}(t)$ will be ultimately bounded ${ }^{7}$ according to*

$$
\lim _{t \rightarrow \infty}\|\underline{e}\| \leq M_{1}
$$

where $M_{1} \rightarrow 0$ as $\sup _{1} \delta_{i} \rightarrow 0$.

In order to show boundedness according to (4.1), we show the existence of a function $V(\underline{e})$ which is positive definite, and a $\dot{V}(\underline{e}, t)$ which is negative for $\|$ e $\|>M_{2}$, where $M_{2} \leq M_{1}, M_{1}$ and $M_{2}$ both being positive numbers. Thereupon, the phase trajectories in the region $\|$ e $\|>M_{2}$ penetrate hyper surfaces $V(\underline{e})=$ const from outside to inside, so that all solutions $\underline{e}(t)$ with bounded initial points are bounded, with an ultimate bound being given by $\|\underline{e}\|=M_{1}$. The need for introducing $M_{1}$ stems from the fact that a trajectory could leave the hyper sphere $\|$ ell $=M_{2}$ and make a bounded excursion out of this region, constrained only by the requirement that $V<0$ at all points on such a trajectory. The hypersphere $\|$ e $\|=M_{1}$ defines the bound on such a motion.

${ }^{*}$ In this paper $\|$ ell denotes the Euclidian norm. 


\section{DESIGN EXAMPLE - CONTROL OF AN EXOTHERMIC CHEMICAL REACTOR}

The problem to be considered is the control of a stirred-tank chemical reactor in which the process may be described as a continuous first-order exothermic chemical reaction. Referring to Fig. 6, the output variables are defined as the temperature $\left(x_{1}^{2}\right)$ of the tank, and concentration ( $\left.x_{1}\right)$ of a particular component (c) of the outflow. The controlled inputs are in turn the flow rate $\left(u_{2}^{*}\right)$ of the coolant, and the inflow concentration ( $u_{1}^{*}$ ) of the component (c). Based on the assumption of ideal mixing, the temperature $\left(\mathrm{x}_{1}^{2}\right)$ and concentration $\left(x_{1}^{l}\right)$ are taken to be uniform throughout the tank. It is desired that $x_{1}^{1}$ and $x_{1}^{2}$ shall be independently controlled.

The equations characterizing the process can be derived from heat and mass balance relationships. Assuming constant volume (inflow = outflow), the heat and mass balance equations can be expressed in normalized form as follows

$$
\begin{aligned}
& \dot{x}_{1}^{1}=u_{1}^{*}-\left(1+k_{1}\left(x_{1}^{2}\right)\right) x_{1}^{1} \\
& \dot{x}_{1}^{2}=k_{1}\left(x_{1}^{2}\right) x_{1}^{1}-\left(x_{1}^{2}-x_{0}\right)\left(1+u_{2}^{*}\right)
\end{aligned}
$$

where

$$
\begin{aligned}
\mathbf{x}_{1}^{1} & =\text { concentration of component } c \text { in outflow } \\
\mathbf{x}_{1}^{2} & =\text { temperature of process } \\
\mathbf{u}_{1}^{*} & =\text { inflow concentration of component } c \\
\mathbf{u}_{2}^{*} & =\text { coolant rate } \\
\mathrm{x}_{0} & =\text { temperature of inflow and coolant } \\
\mathrm{k}_{1}\left(\mathrm{x}_{1}^{2}\right) & =\text { reactor rate term }
\end{aligned}
$$

Using numerical values cited in [8], it will be assumed that

$$
\begin{aligned}
\mathrm{x}_{0} & =1.75 \\
\mathbf{k}_{1}\left(\mathrm{x}_{1}^{2}\right) & =\exp \left(25-50 / \mathrm{x}_{1}^{2}\right)
\end{aligned}
$$

It is observed that the process equations manifest a strong nonlinear intercoupling by virtue of the reaction-rate term $k_{1}\left(x_{1}^{2}\right)$. Further, due to its exothermic nature, the process tends to be open-loop unstable. Thus, for the nominal values of inflow concentration and coolant rate, given respectively by $u_{1}^{*}=1, u_{2}^{*}=1$, the system can be shown to have an unstable equilibrium at $\mathrm{x}_{1}^{1}=0.5, \mathrm{x}_{1}^{2}=2$.

The control problem is to derive a control law which will bring the process states $x_{1}^{1}, x_{1}^{2}$, from an initial point $x_{1}^{1}=0, x_{1}^{2}=1.75$, to a desired operating point $x_{1}^{2}=0.5, x_{1}^{2}=2$. Anticipating that the process will be controlled as a multivariable system, reference is made to the control 
configuration in Fig. 7 wherein the controlled variables $u_{1}$ and $u_{2}$ are defined according to*

$$
\begin{aligned}
& \mathrm{u}_{1}^{*}=\mathrm{U}_{1}+\mathrm{u}_{1}, \\
& \mathrm{u}_{2}^{*}=\mathrm{U}_{2}-\mathrm{u}_{2},
\end{aligned}
$$

$\mathrm{U}_{1}$ and $\mathrm{U}_{2}$ being nominal values.

Following the procedure outlined in section III, we select $A$ and $Q$ to be of the form

$$
\begin{aligned}
& A=\left[\begin{array}{cc}
a_{11}^{1} & 0 \\
0 & a_{11}^{2}
\end{array}\right], \quad a_{11}^{1}<0, a_{11}^{2}<0 \text {, } \\
& Q=\left[\begin{array}{cc}
q_{11}^{1} & 0 \\
0 & q_{11}^{2}
\end{array}\right], \quad q_{11}^{1}>0, q_{11}^{2}>0 .
\end{aligned}
$$

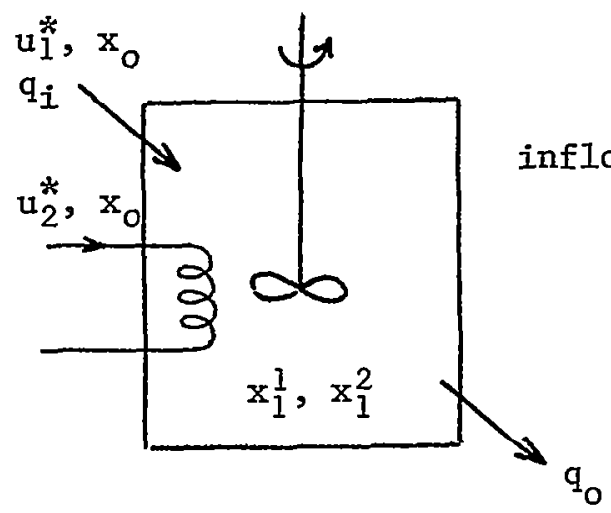

Fig. 6. Stirred-Tank Chemical Reactor

According to (3.5), $P$ in turn takes the form

$$
\mathrm{P}=\left[\begin{array}{cc}
\mathrm{p}_{11}^{1} & 0 \\
0 & \mathrm{p}_{11}^{2}
\end{array}\right] \text {, }
$$

* The equation defining $u_{2}$ is written in such form that $\phi_{22}$ in (3.35) will be positive, as required. 
with $p_{11}^{i}=-q_{11}^{i} / 2 a_{11}^{i}, i=1,2$.

Evaluating the elements $f_{m}^{i}$ in (3.31), it follows, with $U_{1}=U_{2}=1$, that

$$
\begin{aligned}
& f_{1}^{1}=-\dot{s}_{1}^{1}-\left(1+k_{1}\left(x_{1}^{2}\right) x_{1}^{1}+a_{11}^{1} e_{1}^{1}+1+u_{1},\right. \\
& f_{1}^{2}=-\dot{s}_{1}^{2}+k_{1}\left(x_{1}^{2}\right) x_{1}^{1}+a_{i_{1}}^{2} e_{1}^{2}-\left(x_{1}^{2}-x_{0}\right)\left(2-u_{2}\right) .
\end{aligned}
$$

The conditions (3.35), (3.36) which $u_{1}$ and $u_{2}$ must fulfill to guarantee $\dot{v}<0$ will now be obtained. Thus, using (5.8), (3.35) ylelds:

$$
\begin{aligned}
& \left|u_{1}\right| \geq\left|-\dot{s}_{1}^{1}-\left(1+k_{1}\left(x_{1}^{2}\right)\right) x_{1}^{1}+a_{1}^{1} e_{1}^{1}+1\right|, \\
& \left|u_{2}\right| \geq\left|\frac{-\dot{s}_{1}^{2}+k_{1}\left(x_{1}^{2}\right) x_{1}^{1}+a_{1}^{2} e_{1}^{2}-2\left(x_{1}^{2}-x_{0}\right)}{x_{1}^{2}-x_{1}^{1}}\right| .
\end{aligned}
$$

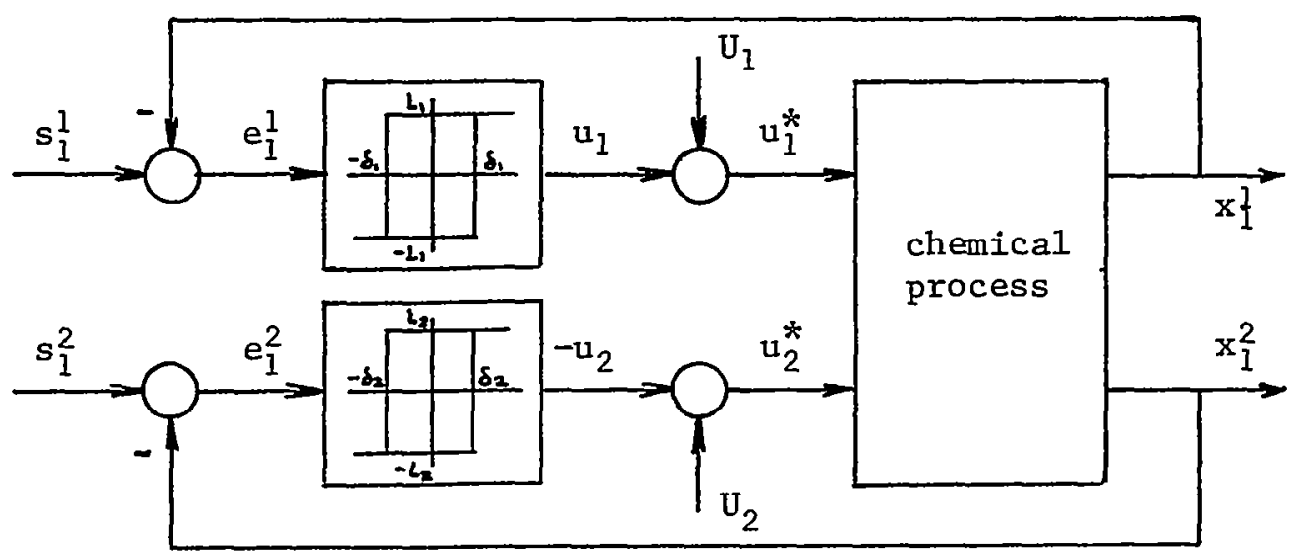

Fig. 7. Multivariable Control Figuration

The equations relating to (3.36) in turn become

$$
\begin{aligned}
& \frac{u_{1}}{\left|u_{1}\right|}=\operatorname{sgn}\left(p_{11}^{I} e_{1}^{1}\right), \\
& \frac{u_{2}}{\left|u_{2}\right|}=\operatorname{sgn}\left(p_{11}^{2} e_{1}^{2}\right) .
\end{aligned}
$$

Noting in Fig. 7 that $\mathrm{u}_{1}$ and $\mathrm{u}_{2}$ are generated by relay controllers, consideration will now be given to the roles of $\mathrm{L}_{1}, \mathrm{~L}_{2}$, and $\delta_{1}, \delta_{2}$ in the design. 
From (3.38) and (5.9) it follows that regions $R_{1}$ and $R_{2}$, in which (5.9) will be satisfied, are defined respectively by

$$
\begin{aligned}
& L_{1} \geq\left|-\dot{s}_{1}^{1}-\left(1+k_{1}\left(x_{1}^{2}\right)\right) x_{1}^{1}+a_{11}^{1} e_{1}^{1}+1\right| \\
& L_{2} \geq\left|\frac{-\dot{s}_{1}^{2}+k_{1}\left(x_{1}^{2}\right) x_{1}^{1}+a_{11}^{2} e_{1}^{2}-2\left(x_{1}^{2}-x_{0}\right)}{x_{1}^{2}-x_{1}}\right| .
\end{aligned}
$$

In this example we shall arbitrarily let $I_{1}=I_{2}=I_{i}$ Furthermore, with reference to $(5.7)$, it is convenient to 1 et $q_{1}^{i}{ }_{1}^{2}=-2 a_{11}^{i}, p_{1}^{i}=1$, in which case it is permissable to let each $a_{1}^{i}$ become arbitrarily small. Hence, the terms $a_{11}^{1} e_{1}^{1}$ and $a_{1}^{2} e_{1}^{2}$ appearing in $(5.11)$ can be ignored. Regions in which control is possible can be determined from (5.11), assuming that $\dot{s}_{1}^{1}$ and $\dot{s}_{1}^{2}$ are negligibly small. The results are plotted in Fig. 8. It may be concluded that multivariable control is possible in the region common to $R_{1}$ and $R_{2}$. Outside of $R_{1}$ and $R_{2}$ the control law is invalid. Inside those portions of $R_{1}$ or $R_{2}$ not common to both, only single-variable control is assured.

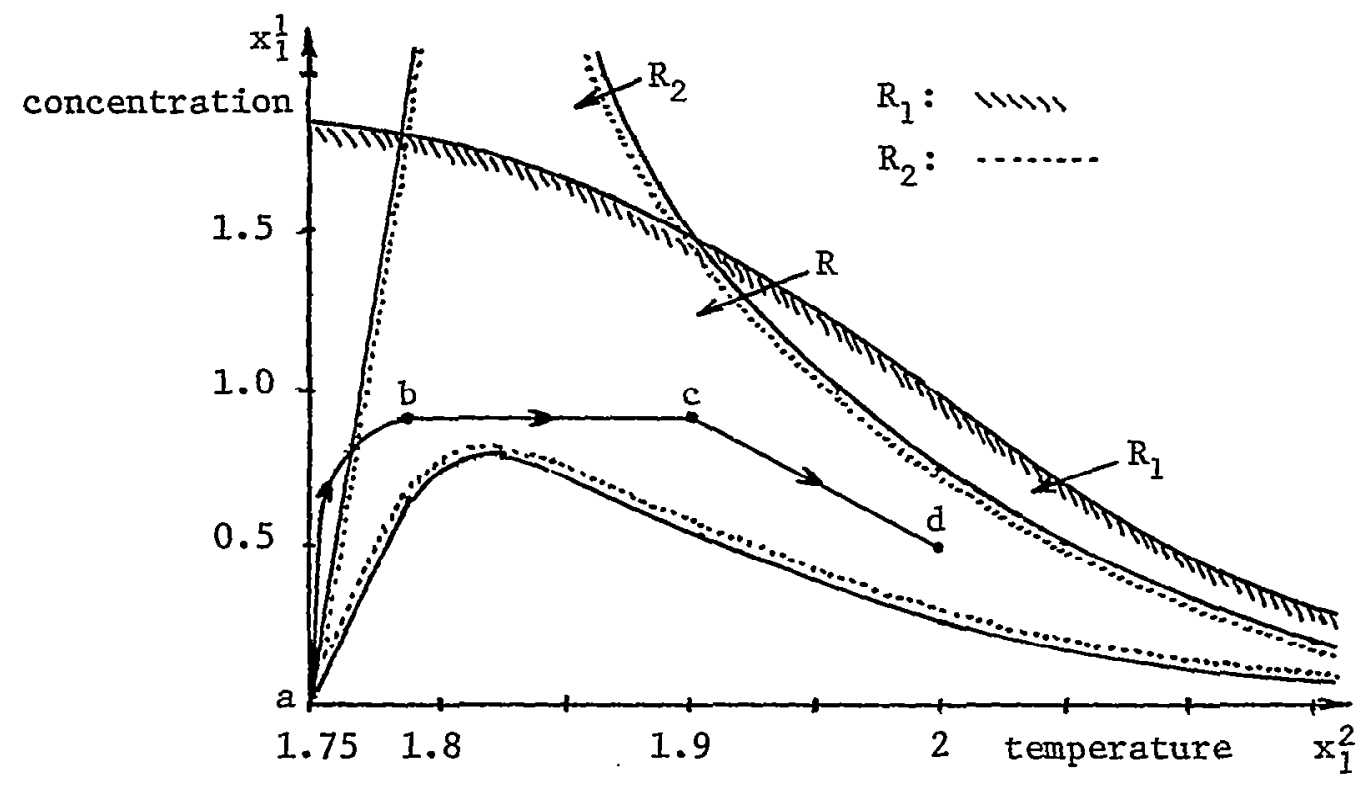

Fig. 8. Regions $R_{1}, R_{2}, R$, Relating to Control of Chemical Reactor

The trajectory shown in Fig. 8 represents one path on which the process variables can be brought from $x_{1}^{1}=0, x_{1}^{2}=1.75$ to $x_{1}^{1}=0.5, x_{1}^{2}=2$. From a to $b$, the process is uncontrolled, with $\mathrm{u}^{*}{ }_{1}=1, \mathrm{u}_{2}^{*}=0$. At point $\mathrm{b}$, the concentration loop is closed and the process is controlled as a single variable system, holding the concentration constant. Thus, from $b$ to $c$, with $\mathrm{u}_{1}^{*}=1+\mathrm{u}_{1}, \mathrm{u}_{2}^{*}=0$, the temperature is allowed to increase without control. 
At point $c$ the temperature loop is closed, and $x_{1}^{1}, x_{1}^{2}$ are controlled simultaneously so as to follow the designated path to point $d$.

The problem we shall concern ourselves with is the motion from $c$ to $d$. According to (5.1I), there are limits which must be set on $\dot{s}_{1}^{1}, \dot{s}_{1}^{2}$, in order that the control law shall be valid. Within these limits, a bound on the error can be found which depends upon $\delta_{1}$ and $\delta_{2}$. In this problem it is readily seen that $\dot{v}_{i}<0$ for $\left|e_{1}^{i}\right| \geq \delta_{i}$, whereas for $\left|e_{1}^{i}\right|<\delta_{i}$, the sign of $v_{i}$ is not assured of being negative. Hence, $\delta_{i}$ is seen to be an ultimate bound on $e_{1}^{i}$, for $i=1,2$. Let us assume that $\delta_{1}=\delta_{2}=0.01$.

As noted in Section III, a bound on $\left|\dot{s}_{1}^{1}\right|$ and $\left|\dot{s}_{1}^{2}\right|$ could be instrumented in a model (Fig. 4) to assure the validity of (5.11) along the designated path. A more efficient solution to the problem would be to compute the maximum values of $\left|\dot{s}_{1}^{1}\right|,\left|\dot{s}_{1}^{2}\right|$, which are permitted at each point along the path. This approach is taken here.

According to the slope of the trajectory from $c$ to d, it is seen that $\dot{s}_{1}^{1}<0, \dot{s}_{1}^{2}>0$. Thus, from (5.11) we solve for $\left|\dot{s}_{1}^{1}\right|_{\max }$ and $\left|\dot{s}_{1}^{2}\right|_{\max }$ according to the equations

$$
\begin{aligned}
& \left|\dot{s}_{1}^{1}\right|_{\text {max }}=\left(1+k_{1}\left(x_{1}^{2}\right) x_{1}^{1}\right) \\
& \left|\dot{s}_{1}^{2}\right|_{\max }=k_{1}\left(x_{1}^{2}\right) x_{1}^{1}-\left(x_{1}^{2}-x_{0}\right)
\end{aligned}
$$

The slope of the trajectory imposes the constraint $\left|\dot{s}_{1}^{1}\right|=4\left|\dot{s}_{1}^{2}\right|$. Hence, the validity of (5.11) is preserved by the following statement:

$$
\begin{aligned}
& \text { if } \frac{\left|\dot{s}_{1}^{1}\right|_{\max }}{\left|\dot{s}_{1}^{2}\right|_{\max }} \leq 4 \text {, use }\left|\dot{s}_{1}^{2}\right|_{\max } \\
& \text { if } \frac{\left|\dot{s}_{1}^{1}\right|_{\max }}{\left|\dot{s}_{1}^{2}\right|_{\max }}>4 \text {, use }\left|\dot{s}_{1}^{1}\right|_{\max } \text {. }
\end{aligned}
$$

Data based on digital computation is presented in Table I, showing the errors incurred in tracking motions along the trajectory cd. In Table I-a the velocity was computed as above. In Table $I-b$ the velocity was computed to have twice the magnitude permitted in order for (5.11) to be valid. Allowing for small discrepancies in the error bound as a result of discretization, it is observed that the errors in (a) are within the bounds allowed by the synthesis procedure, whereas in (b) this bound in $e_{1}^{2}$ is clearly violated. 


\begin{tabular}{|c|c|c|c||c|c|c|c|}
\hline \multicolumn{9}{|c|}{$a$} & \multicolumn{5}{|c|}{$b$} \\
\hline$s_{1}^{1}$ & $x_{1}^{1}$ & $s_{1}^{2}$ & $x_{1}^{2}$ & $s_{1}^{1}$ & $x_{1}^{1}$ & $s_{1}^{2}$ & $x_{1}^{2}$ \\
\hline 0.90 & 0.88 & 1.90 & 1.90 & 0.90 & 0.89 & 1.90 & 1.90 \\
0.86 & 0.88 & 1.91 & 1.90 & 0.83 & 0.84 & 1.92 & 1.90 \\
0.83 & 0.85 & 1.92 & 1.91 & 0.77 & 0.77 & 1.93 & 1.91 \\
0.80 & 0.80 & 1.93 & 1.92 & 0.71 & 0.71 & 1.95 & 1.92 \\
0.75 & 0.75 & 1.94 & 1.93 & 0.65 & 0.66 & 1.96 & 1.92 \\
0.70 & 0.71 & 1.95 & 1.94 & 0.60 & 0.61 & 1.98 & 1.93 \\
0.64 & 0.65 & 1.96 & 1.95 & 0.55 & 0.54 & 1.99 & 1.94 \\
0.59 & 0.61 & 1.98 & 1.97 & 0.50 & 0.51 & 2.0 & 1.94 \\
0.51 & 0.52 & 2.0 & 1.98 & 0.50 & 0.50 & 2.0 & 1.95 \\
0.50 & 0.49 & 2.0 & 1.99 & 0.50 & 0.50 & 2.0 & 1.95 \\
0.50 & 0.50 & 2.0 & 1.99 & 0.50 & 0.50 & 2.0 & 1.96 \\
\hline
\end{tabular}

Increments shown for intervals of 0.1 time units

Table I

VI. CONCLUSIONS

The design of control systems based on the linear approximation fails to predict system behavior for large perturbations about the operating point. Furthermore, in the case of strongly nonlinear systems, particularly of the multivariable variety, the value of classical design techniques is greatly diminished. To cope with this problem a design technique has been developed which treats the nonlinear equations of the plant directly. The presence of hard-saturation constraints on the controlled forces is taken into account by establishing a region in which the plant can be controlled. The size of this region is in turn related to certain characteristics of the command inputs to the plant. By means of a nonlinear model, or a computational procedure, it is then possible to develop a set of plant inputs which the plant is capable of following with arbitrarily small error, provided the plant state vector is held within a specified region.

Because the control law generally satisfies only a sufficiency condition for boundedness of the error, it is to be expected that the design may be 
conservative. For example, the region $\mathrm{R}$, inside which control is assured, may be unnecessarily restrictive. This criticism should, however, be weighed against the fact that a design which depends upon linearization fails to specify a region in which the design is valid. The convenient and commonly adopted assumption that a plant can be adequately represented by a linear part preceded by a nonlinearity is perhaps not worthy of as much attention as it has received. It is hoped that this paper will serve to demonstrate the advantages to be gained by working with the nonlinear differential equations. 


\section{APPENDIX I. DERIVATION OF AN ULTIMATE ERROR BOUND}

It will be shown that, with each $u_{i}$ in (2.1) governed by (3.37), and with the plant state ( $x$ ) restricted to some region $\mathrm{R}$, a control law can be found which causes the solution of $(3.2)$ to have an ultimate bound according to

$$
\lim _{t \rightarrow \infty} \| \text { ell } \leq M_{1}
$$

where $M_{1} \rightarrow 0$ as sup $\delta_{i} \rightarrow 0$. This result can be derived without restricting the $P$ matrix in (3.11) to have the partitioned diagonal form of (3.8). However, it will serve the needs of this paper, and simplify the derivation, to assume here that $P$ conforms to $(3.8)$.

As noted in Section IV, to prove boundedneșs, it is sufficient to show, for some positive definite function $\mathrm{V}(\underline{e})$, that $\mathrm{V}(\underline{e}, t)<0$ outside of some region $\|$ ell $>M_{2}, M_{2} \leq M_{1}$. According to (3.16) through (3.19), it suffices, with $\mathrm{P}$ in the form of $(3.8)$, to show that $\dot{V}_{i}(\underline{e}, t)<0$ for $\left\|\underline{e}_{i}\right\|>M_{2}^{i}, i=1$, ..., q, since each $V_{i}$ is positive definite.

From (3.19), (3.30), $\dot{\mathrm{V}}_{\mathbf{i}}$ can be written as

$$
\dot{v}_{i}=-\underline{e}_{i}^{t} \underline{e}_{i}-\gamma_{i} f_{m}^{i}
$$

where for simplicity we have chosen $Q_{i i}$ as the unit matrix. According to $(3.31),(3.32)$,

$$
f_{m}^{i}=-s_{m}^{i}+g_{m}^{i}(\underline{x}, t)+\sum_{j=1}^{m} a_{m j}^{i} e_{j}^{i}+\sum_{j=1}^{q} \phi_{m j}(\underline{x}, t) u_{j} \cdot
$$

We shall assume that all terms in $f_{m}^{i}$ are appropriately bounded, including $\left\|e_{i}\right\| \leq M_{i}^{i}$, and that an appropriate region $R_{i}$ in $x$ space has been determined so that, for $\left|\gamma_{i}\right| \geq \delta_{i}$, both (3.35) and (3.36) are satisfied and $\dot{v}_{i}<0$. It must now be shown that there is a $\delta_{i}$ such that, with $\left|\gamma_{i}\right|<\delta_{i}$, the inequality $\dot{\mathrm{V}}_{i}<0$ prevails for some $\left\|\underline{e}_{i}\right\|>\mathrm{M}_{2}^{\frac{1}{1}}$, where $\mathrm{M}_{2}^{\dot{1}} \leq \mathrm{M}_{\dot{j}}^{\dot{1}}$. It will be recalled that $\left|\gamma_{i}\right|<\delta_{i}$ defines the condition for which $(3.35),(3.36)$ can fail to be satisfied.

From (A.2) we have the inequality

$$
\dot{v}_{i} \leq-e_{i}^{t} e_{i}+\left|r_{i}\right|\left|f_{m}^{i}\right| \text {. }
$$

For the condition $\left|\gamma_{i}\right|<\delta_{i}$, it follows that

$$
\dot{\mathrm{v}}_{i} \leq-e_{i}^{t} e_{i}+\delta_{i}\left|f_{m}^{i}\right| \text {. }
$$


Using

$$
\begin{aligned}
\sum_{j=1}^{m} a_{m j}^{i} e_{j}^{i} & \leq \operatorname{supp}_{j}\left|a_{m j}^{i}\right| \sum_{j=1}^{m}\left|e_{j}^{i}\right| \\
& \left.\leq \operatorname{supp}_{j}\left|a_{m j}^{i}\right|{ }_{(m)}^{m}\left(e_{j}^{i}\right)^{2}\right)^{l / 2} \\
& \leq k_{I}^{i}|| e_{i} \|,
\end{aligned}
$$

it is possible to write in place of (A.5)

$$
\dot{\mathrm{v}}_{i} \leq-\left\|\underline{e}_{i}\right\|^{2}+\delta_{i}\left|k_{1}^{i}\right| \underline{e}_{i} \|+k_{2}^{i} \mid
$$

where $k \frac{i}{2}$ is an upper bound on the magnitude of the sum of all terms in $f_{m}^{i}$ not involving components of $\underline{e}_{i}$. For the assumed bound $M_{1}^{i}$ there is always a $\delta_{i}$ which is small enough so that in (A.6), with $\left\|\underline{e}_{i}\right\|>M_{2}^{i}, \dot{V}_{i}<0$. The value of $\mathrm{M}_{2}^{i}$, required to yield the ultimate bound $M_{1}^{i}$, depends on the particular $p_{i i}$ matrix.

It follows directly that, if each $e_{i}$ is ultimately bounded, then $\underline{e}$ is bounded according to $A .1$, where $\left(M_{1}\right)^{2}=\sum_{i=1}^{q}\left(M_{1}^{l}\right)^{2}$. Since each $M_{1}^{i} \rightarrow 0$ as $\delta_{i} \rightarrow 0$, it follows that $M_{1} \rightarrow 0$ as $\sup _{i} \delta_{i} \rightarrow 0$. $i=1$

\section{REFERENCES}

[1] Grayson, L. P., "Design via Liapunov's Second Method," Preprints, Fourth J.A.C.C., Minneapolis, Minnesota, 1963, pp. 589-595.

[2] Monopoli, R. V., "Synthesis Techniques Employing the Direct Method," I.E.E.E. AC-10.3, 1965, pp. 369-370.

[3] Lindorff, D. P. and R. V. Monopoli, "Control of Time Variable Nonlinear Multivariable Systems Using Liapunov's Direct Method," Preprints, Seventh J.A.C.C., Seattle, Washington, 1966, pp. 475-484.

[4] Monopoli, R. V. and L. P. Grayson, "Discussion on Two Theorems on the Second Method," T.E.E.E. AC-11.1, 1966, PP. 140-141.

[5] Hahn, W., "Theory and Application of Liapunov's Direct Method," PrenticeHa11, Englewood Cliffs, New Jersey, 1963.

[6] Taylor, T. M., "Effect of Transducer Noise on Design of a Controller for Nonautonomous Plants," M.S. Thesis, University of Connecticut, Storrs, Connecticut, 1966.

[7] Hahn, w., loc. cit., p. 129.

[8] Aris, R. and N. Amundson, "On Analysis of Chemical Reactor Stability and Control" - I and II, Chem. Eng. Sc., 7.3, 1958, pp. 121-147. 